\title{
Polarimetric subspace target detector for SAR data based on the Huynen dihedral model
}

\author{
Vic Larson \\ George Mason University \\ C3I Center \\ Fairfax, VA 22030
}

\author{
Les Novak \\ MIT Lincoln Laboratory \\ Lexington, MA 02173
}

\begin{abstract}
Two new polarimetric subspace target detectors are developed based on a dihedral signal model for bright peaks within a spatially extended target signature. The first is a coherent dihedral target detector based on the exact Huynen model for a dihedral. The second is a noncoherent dihedral target detector based on the Huynen model with an extra unknown phase term. Expressions for these polarimetric subspace target detectors are developed for both additive Gaussian clutter and more general additive spherically invariant random vector (SIRV) clutter including the $K$-distribution. For the case of Gaussian clutter with unknown clutter parameters, constant false alarm rate (CFAR) implementations of these polarimetric subspace target detectors are developed. The performance of these dihedral detectors is demonstrated with real millimeter-wave fully polarimetric SAR data. The coherent dihedral detector which is developed with a more accurate description of a dihedral offers no performance advantage over the noncoherent dihedral detector which is computationally more attractive. The dihedral detectors do a better job of separating a set of tactical military targets from natural clutter compared to a detector that assumes no knowledge about the polarimetric structure of the target signal.
\end{abstract}

\section{INTRODUCTION}

The goal of this work is to detect non-moving military vehicles using high resolution, millimeter-wave, multi-polarimetric channel, synthetic aperture radar (SAR) data. The SAR scenes in which we wish to find these targets contain natural clutter (such as grass, crops, shrubs, trees, and rocks) and manmade clutter (including for example, roads, buildings, and vehicles). Some of the manmade clutter, especially vehicles and buildings, can look very much like the targets of interest. The targets we wish to detect have a spatially extended signal that is very dependent on the unknown orientation of the target in the scene and multi-path from surrounding clutter. Much of the energy of the spatially extended targets is localized in bright single pixel returns caused by corner reflectors, primarily dihedrals and trihedrals on the target. Dihedrals have an even bounce polarimetric signal whereas trihedrals have an odd bounce polarimetric signal. It has been previously shown that military targets exhibit equal amounts of odd bounce and even bound scattering whereas natural clutter exhibits primarily odd bounce scattering. ${ }^{1}$ This research involves developing two new target detectors that will separate military targets from most of the natural clutter using the bright single pixel dihedral peaks (or even bounce scattering) within the spatially extended target signature. The rejection of most of the manmade clutter that can look very much like the targets is left for subsequent higher level processing stages not covered in this paper. ${ }^{2}$

A thresholding operation or simple single pixel, single polarimetric channel constant false alarm rate (CFAR) detector will separate bright manmade objects (including the targets) from the dimmer natural clutter in SAR data; however, these detectors suffer from a high number of false alarms caused by speckle or coherent noise in the SAR image. ${ }^{2}$ For a millimeter-wave SAR which gets a strong signal from the tree canopy, an especially high number of false alarms come from tree canopies.

With a fully polarimetric SAR system we have three polarimetric channels available to detect targets and reject clutter. One of the most robust approaches for polarimetric SAR target detection is the polarimetric whitening filter (PWF) target detector first developed by Novak. ${ }^{3-8}$ This detector makes no assumption about the polarimetric structure of the target signal and optimally reduces the speckle or coherent noise to enhance detection performance. Novak et. al. have shown that the PWF target detector performs better than polarimetric target detectors based on simple deterministic and random signal models using real millimeter-wave SAR data. ${ }^{3}$

In this research, two new polarimetric target detectors are developed based on modeling the target as deterministic with unknown parameters. The dominant scatterers from the vehicles in our target set exhibit both dihedral and trihedral polarimetric characteristics, however the dominant scatterers from the natural clutter in our data exhibit more trihedral or flat plate polarimetric characteristics. Using the Huynen polarimetric scatterer model ${ }^{9}$, two new detectors are developed for the 
dihedrals in a target signature which do a good job of rejecting unstructured clutter and discrete clutter with trihedral or flat plate scattering. The first is a noncoherent approach which leads to an especially simple detector. The second is a more complicated coherent detector which is based on the exact Huynen model for a dihedral. For the case where the clutter parameters are known, we derive these detectors for the target signal in the presence of additive spherically invariant random vector (SIRV) clutter. ${ }^{10-12}$ The SIRV clutter model is a general parametric clutter model for correlated vector data which is not necessarily Gaussian. The Gaussian distribution is a special case of the SIRV clutter model. The $K$-distribution, which has been widely used to model radar clutter, is also a special case of the SIRV model.

Other researchers have considered using the Huynen model as part of a target detection scheme. De Graaf developed matched filter and quadratic target detectors using the Huynen model. ${ }^{13}$ Cameron and Leung developed a technique to decompose a canonical Huynen scatterer without regard to clutter. ${ }^{14}$ Dilsavor and Moses developed a polarimetric target detector for dihedrals and other scatterers in additive Gaussian and $K$-distributed clutter using the general Huynen model with unknown nonrandom parameters. The Dilsavor-Moses detector for the case of a dihedral scatterer does not have a closed form expression and includes a 1-D search. ${ }^{15,16}$ Our new dihedral detector, which is also based on the Huynen signal model with unknown parameters in additive clutter, has an explicit closed form expression and is shown to be very effective as a SAR target detector. Michael B. Sechtin of MIT Lincoln Laboratory developed a dihedral whitening filter detector (DIWF) which is the PWF combination of the two polarimetric channels that respond best to a dihedral based on the Huynen model (the $H V$ and $H H-V V$ channels).$^{17}$ Our dihedral detector has performance similar to the DIWF detector, but is derived more rigorously. The DIWF detector algorithm is presented in the Appendix. Our dihedral detector is also related to the work of Kelly on radar target detection. ${ }^{18,19}$ In fact our adaptive noncoherent dihedral detector in Gaussian clutter is a special case of a more general radar target detection framework developed by Kelly and Forsythe. ${ }^{19}$ This work is related to the radar target detection work of Reed, Anderson, and Yu who developed a multi-pixel spatial subspace SAR target detector for polarimetric SAR data where the target is represented with arbitrary spatial basis functions. ${ }^{20-22}$ This new dihedral detector is a single pixel, multi-polarimetric channel target detector which makes use of a target subspace in the non-white polarimetric dimension. Our dihedral detector is also tied to a known physical polarimetric target signal model (the Huynen model), includes a coherent formulation, and for the non-CFAR case is derived for general additive SIRV clutter.

This paper is organized in the following manner. In Section 2, two new polarimetric subspace target detectors are derived using the SIRV clutter model. The form of the detectors for the special case of Gaussian clutter and the dihedral target model are shown explicitly. Section 3 contains the development of constant false alarm rate (CFAR) detectors for the special case of Gaussian clutter. In Section 4, we present performance results demonstrating these new SAR target detectors against real polarimetric SAR data.

\section{NONADAPTIVE POLARIMETRIC SUBSPACE TARGET DETECTOR}

In this Section, the polarimetric subspace target detectors are derived under the assumption that the clutter parameters are known. In Section 3, these detectors are rederived for the case where the clutter has unknown parameters. Two polarimetric subspace target detectors are derived in this Section. The first derivation is for a noncoherent subspace (i.e. the linear basis functions representing the target signal each have independent unknown phase). The second derivation is for a coherent subspace (i.e., the linear basis functions representing the target signal share a common unknown phase). The noncoherent approach leads to a simpler test with little performance loss as compared with the coherent test.

These detectors are derived for the general case of non-Gaussian SIRV clutter using the same approach as Sangston and Gerlach. ${ }^{12}$ The derivation for SIRV clutter is not much more complicated than the derivation for Gaussian clutter and leads to a more general result. It is also shown for the noncoherent detector that when the target signal basis functions span the entire polarimetric space, the detector is equivalent to the PWF detector.

\subsection{Problem formulation}

The detection problem solved here is for a signal with unknown parameters in additive SIRV clutter with known parameters. There are two hypotheses, the target present hypothesis $\left(H_{l}\right)$ and the clutter only hypothesis $\left(H_{0}\right)$.

$$
\begin{aligned}
& H_{1}: \mathbf{x}=\mathbf{x}_{s}(A, \phi, \psi)+\tau \cdot \mathbf{x}_{c} \\
& H_{0}: \mathbf{x}=\tau \cdot \mathbf{x}_{c}
\end{aligned}
$$


The signal vector, $\boldsymbol{x}$, is a polarimetric vector for a single pixel in the SAR scene and has the following form:

$$
\mathbf{x}=\left[\begin{array}{l}
H H \\
H V \\
V V
\end{array}\right]=\left[\begin{array}{l}
H H_{I}+j H H_{Q} \\
H V_{I}+j H V_{Q} \\
V V_{I}+j V V_{Q}
\end{array}\right]
$$

where $H H$ is the horizontal transmit/horizontal receive channel, $H V$ is the horizontal transmit/vertical receive channel, and $V V$ is the vertical transmit/vertical receive channel. $I$ denotes the in-phase channel and $Q$ denotes the quadrature channel. The inphase and quadrature channels are uncorrelated and have equal variance, so this data can be modeled using the complex circular symmetric model. ${ }^{10}$ By the reciprocity theorem, the $V H$ channel has the same statistics as the $H V$ channel, so the $V H$ channel is not used. ${ }^{3-5}$ The clutter signal is additive with respect to the target signal, and the clutter signal is the product of a positive independent scalar random variable, $\tau$, with probability density function (pdf), $f_{\tau}(\tau)$, and a zero mean complex Gaussian signal with covariance, $\Sigma_{\mathrm{c}}$. The covariance has a specific structure because the $H H$ and $H V$ channels are physically uncorrelated, and the $V V$ and $H V$ channels are physically uncorrelated. We use the same notation as Novak to express the nonzero parameters of the polarimetric covariance: ${ }^{3-5}$

$$
\Sigma_{c}=\sigma_{H H} \cdot\left[\begin{array}{ccc}
1 & 0 & \rho \sqrt{\gamma} \\
0 & \varepsilon & 0 \\
\rho^{*} \sqrt{\gamma} & 0 & \gamma
\end{array}\right]
$$

where * denotes complex conjugate. The SIRV clutter is fully specified by the covariance $\left(\Sigma_{\mathrm{c}}\right)$ of the vector Gaussian term and the pdf $\left(f_{\tau}(\tau)\right)$ of the scalar multiplicative term. The second moment of the scalar random variable is constrained to be one (i.e., $\left.E\left\{\tau^{2}\right\}=1\right)$ so that the covariance of $\boldsymbol{x}_{c}$ is $\Sigma_{c}$ not $E\left\{\tau^{2}\right\} \Sigma_{c}{ }^{10}$ The pdf of $\boldsymbol{x}$ can be found in terms of the pdf of $\tau$ and the pdf of $\boldsymbol{x}_{c}$.

$$
\begin{aligned}
& f_{x}\left(\mathbf{x} \mid H_{0}\right)=\int_{0}^{\infty} f_{x}\left(\mathbf{x} \mid H_{0}, \tau\right) f_{\tau}(\tau) d \tau \\
& f_{x}\left(\mathbf{x} \mid H_{0}\right)=\frac{1}{\pi^{N}\left|\Sigma_{c}\right|} \int_{0}^{\infty} \frac{1}{\tau^{N}} \cdot \exp \left(\mathbf{x}^{H} \Sigma_{c}^{-1} \mathbf{x} / \tau^{2}\right) f_{\tau}(\tau) d \tau
\end{aligned}
$$

Different SIRV clutter models can be used by specifying a different scalar pdf $\left(f_{\tau}(\tau)\right)$. We use both the Gaussian and $K$ distribution which are specified as follows: ${ }^{10}$

$$
\begin{aligned}
& f_{\tau}(\tau)=\delta(\tau-1) \Rightarrow f_{x}\left(\mathbf{x} \mid H_{0}\right)=\frac{1}{\pi^{N}\left|\Sigma_{c}\right|} F_{N}(p) \text { where } p=\mathbf{x}^{H} \Sigma_{c}^{-1} \mathbf{x} \text { and } F_{N}(p)=\exp (-p) \\
& f_{\tau}(\tau)=\frac{2\left(v \tau^{2}\right)^{v} \exp \left(-v \tau^{2}\right)}{\tau \Gamma(v)} \Rightarrow f_{x}\left(\mathbf{x} \mid H_{0}\right)=\frac{1}{\pi^{N}\left|\Sigma_{c}\right|} F_{N}(p) \text { where } p=\mathbf{x}^{H} \Sigma_{c}^{-1} \mathbf{x} \text { and } F_{N}(p)=\frac{2 v^{N}}{\Gamma(v)}(\sqrt{v p})^{v-N} K_{N-v}(2 \sqrt{v p})
\end{aligned}
$$

where the $f_{\tau}(\tau)$ that gives Gaussian clutter is an impulse at one and the $f_{\tau}(\tau)$ that gives $K$-distributed clutter is the pdf for the square root of the Gamma random variable constrained so that $E\left\{\tau^{2}\right\}=1 .{ }^{10}$ Other SIRV clutter models can be developed using other scalar distributions $\left(f_{\tau}(\tau)\right){ }^{10-12}$ The only restriction is that the resulting SIRV pdf $\left(f_{x}(\boldsymbol{x})\right)$ must be a valid pdf for all $N$.

The signal model is deterministic with unknown nonrandom parameters. We use the Huynen model to represent the polarimetric signal for a dihedral scatterer: ${ }^{9}$

$$
\mathbf{x}_{s}(A, \phi, \psi)=\left[\begin{array}{c}
H H \\
H V \\
V V
\end{array}\right]=A e^{j \phi}\left[\begin{array}{c}
\cos (2 \psi) \\
\sin (2 \psi) \\
-\cos (2 \psi)
\end{array}\right]
$$

The target signal model can also be approximately represented as a sum of linear basis functions, $\mathbf{V}$, with unknown complex weights, $\alpha$. This approach of representing a radar signal with a set of linear basis functions was used by Reed, Anderson, and $\mathrm{Yu}$ to represent a radar target signal in the spatial domain. ${ }^{20-22}$ We use the same approach to represent the radar signal in the polarimetric domain. 


$$
\begin{aligned}
& \mathbf{x}_{s}(\alpha)=\left[\begin{array}{c}
H H \\
H V \\
V V
\end{array}\right]=\sum_{i=1}^{M} \alpha_{i} \mathbf{v}_{i}=\mathbf{V} \alpha \quad \text { where } \quad \mathbf{V}=\left[\begin{array}{lll}
\mathbf{v}_{1} & \cdots & \mathbf{v}_{M}
\end{array}\right] \\
& \alpha=\left[\begin{array}{c}
\alpha_{1} \\
\vdots \\
\alpha_{M}
\end{array}\right] \\
& \mathbf{v}_{i} \text { is } N x 1 \text { known basis vector } \\
& \alpha_{i} \text { is unknown complex scalar }
\end{aligned}
$$

The number of basis functions, $M$, is less than or equal to the dimensionality of the data, $N$. In the case of polarimetric data used in this paper, $N=3$, so $M \leq 3$. The work in this paper focuses on single frequency multi-polarimetric channel data. These same techniques could be applied to multi-frequency, multi-polarimetric channel data which is higher dimensional data (i.e., $N>3$ ).

This noncoherent signal model can be used to approximately represent a dihedral using the following linear basis functions:

$$
\mathbf{x}_{s}=\alpha_{1}\left[\begin{array}{c}
1 \\
0 \\
-1
\end{array}\right]+\alpha_{2}\left[\begin{array}{l}
0 \\
1 \\
0
\end{array}\right]
$$

This dihedral model obviously does not match the Huynen model because each of the basis vectors has an independent phase and there are four unknown parameters (the real and imaginary parts of $\alpha_{1}$ and $\alpha_{2}$ ) instead of the three parameters $(A, \phi$, and $\psi$ ) represented in the Huynen model. The unknown parameters of the linear basis functions are in rectangular coordinates whereas the Huynen model uses polar coordinates. Since the unknown parameters are nonrandom, any coordinate system can be used to represent the unknown space.

The phase discrepancy between Equation 8 and the Huynen dihedral model of Equation 6 motivates a coherent polarimetric subspace model which is used in the derivation of the coherent polarimetric subspace target detector. For the coherent subspace model, there is a single unknown phase, $\phi$, and each known basis function, $\mathbf{v}_{i}$, has an unknown real scalar, $\alpha_{R i}$.

$$
\begin{aligned}
& \mathbf{x}_{s}=e^{j \phi} \sum_{i=1}^{M} \alpha_{R i} \mathbf{v}_{i}=e^{j \phi} \mathbf{V} \alpha_{R} \quad \text { where } \mathbf{V}=\left[\begin{array}{lll}
\mathbf{v}_{1} & \cdots & \mathbf{v}_{M}
\end{array}\right] \\
& \alpha_{R}=\left[\begin{array}{c}
\alpha_{R 1} \\
\vdots \\
\alpha_{R M}
\end{array}\right] \\
& \mathbf{v}_{i} \text { is } N x 1 \text { known basis vector } \\
& \alpha_{R i} \text { is unknown real scalar } \\
& \phi \text { is unknown absolute phase }
\end{aligned}
$$

This model exactly matches the Huynen dihedral model where again the unknown parameters are in rectangular coordinates $\left(\alpha_{R 1}, \alpha_{R 2}\right)$ as opposed to the polar coordinates $(A, \psi)$ of the Huynen model.

$$
\begin{aligned}
& \mathbf{x}_{s}=e^{j \phi}\left\{\alpha_{R 1}\left[\begin{array}{c}
1 \\
0 \\
-1
\end{array}\right]+\alpha_{R 2}\left[\begin{array}{l}
0 \\
1 \\
0
\end{array}\right]\right\} \\
& \alpha_{R 1}=A \cos 2 \psi \\
& \alpha_{R 2}=A \sin 2 \psi
\end{aligned}
$$

Both the rectangular and polar coordinates specify the dihedral amplitude subspace. We again emphasize that is equally valid to solve for the detector using either set of coordinates since the unknown parameters are nonrandom. 


\subsection{Derivation of noncoherent polarimetric subspace target detector}

The dihedral detectors are derived using the generalized likelihood ratio test (GLRT) approach ${ }^{33}$ where each hypothesis is maximized over the unknown parameters in the likelihood ratio test. The GLRT derivation of these detectors is solved using the same technique as Sangston and Gerlach. ${ }^{12}$ For the noncoherent polarimetric subspace detector, the GLRT approach leads to the following expression for the likelihood function:

$$
\begin{gathered}
\frac{\max _{\alpha} f_{x}\left(\mathbf{x} \mid H_{1}, \alpha\right)}{f_{x}\left(\mathbf{x} \mid H_{0}\right)}=\frac{\max _{\alpha} F_{N}\left((\mathbf{x}-\mathbf{V} \alpha)^{H} \Sigma_{c}^{-1}(\mathbf{x}-\mathbf{V} \alpha)\right)}{F_{N}\left(\mathbf{x}^{H} \Sigma_{c}^{-1} \mathbf{x}\right)} \underset{H_{0}}{H_{1}} \eta \\
\text { where, } F_{N}(p)=\int_{0}^{\infty} \frac{1}{\tau^{N} e^{-\left(p / \tau^{2}\right)}} f_{\tau}(\tau) d \tau
\end{gathered}
$$

Equation 11 is obtained by evaluating the pdf of each hypothesis of Equation 1 and inserting each pdf into the GLRT. For the specific case of Gaussian clutter, $F_{N}(p)=e^{-p}$, and the expression simplifies to a ratio of exponentials maximized over $\alpha$. To simplify Equation 11, the maximization of the target pdf over the unknown parameter, $\alpha$ must be solved. This solution is found by setting the first derivative to zero.

$$
\begin{gathered}
\max _{\alpha} F_{N}\left((\mathbf{x}-\mathbf{V} \alpha)^{H} \Sigma_{c}^{-1}(\mathbf{x}-\mathbf{V} \alpha)\right)=\max _{\alpha} F_{N}(p) \text { where } p=(\mathbf{x}-\mathbf{V} \alpha)^{H} \Sigma_{c}^{-1}(\mathbf{x}-\mathbf{V} \alpha) \\
\frac{\partial F_{N}}{\partial \alpha}=\left.\frac{d F_{N}}{d p} \cdot \frac{\partial p}{\partial \alpha}\right|_{\alpha=\hat{\alpha}}=\mathbf{0}
\end{gathered}
$$

The derivative of $F_{N}$ with respect to $p$ is the following:

$$
\frac{d F_{N}}{d p}=-\int_{0}^{\infty} \frac{1}{\tau^{N+2}} e^{-\left(p / \tau^{2}\right)} f_{\tau}(\tau) d \tau
$$

since $F_{N}(p)$ is monotonically decreasing by the definition of the SIRV clutter model, ${ }^{10-12}$ it follows that $\frac{d F_{N}}{d p}<0$ and the solution must come from $\frac{\partial p}{\partial \alpha}=\mathbf{0}$.

$$
\begin{aligned}
& \frac{\partial p}{\partial \alpha}=-\mathbf{V}^{H} \Sigma_{c}^{-1} \mathbf{x}+\mathbf{V}^{H} \Sigma_{c}^{-1} \mathbf{V} \alpha=\mathbf{0} \\
& \hat{\alpha}=\left(\mathbf{V}^{H} \Sigma_{c}^{-1} \mathbf{V}\right)^{-1} \mathbf{V}^{H} \Sigma_{c}^{-1} \mathbf{x}
\end{aligned}
$$

Now the final form of the GLRT detector can be expressed in terms of the maximum likelihood estimate of $\alpha$.

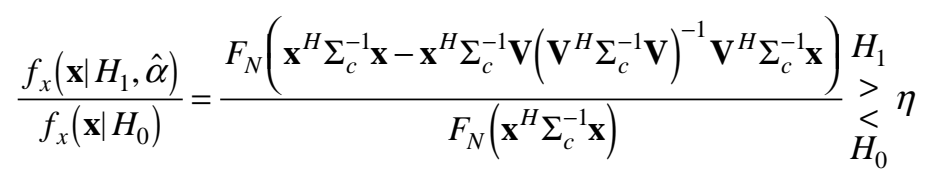

This expression of the detector can be simplified by using the $N-M$ basis vectors defining the subspace that is orthogonal to the target subspace through the clutter whitening operation:

$$
\begin{aligned}
& \mathbf{U}=\left[\begin{array}{llll}
\mathbf{u}_{1} & \mathbf{u}_{2} & \cdots & \mathbf{u}_{N-M}
\end{array}\right] \\
& \text { where } \mathbf{U}^{H} \Sigma_{c}^{-1} \mathbf{V}=\mathbf{0}_{(N-M) x M}
\end{aligned}
$$

Equation 17 can be rewritten in terms of $\boldsymbol{U}$ as: 


$$
\frac{f_{x}\left(\mathbf{x} \mid H_{1}, \hat{\alpha}\right)}{f_{x}\left(\mathbf{x} \mid H_{0}\right)}=\frac{F_{N}\left(\mathbf{x}^{H} \Sigma_{c}^{-1} \mathbf{U}\left(\mathbf{U}^{H} \Sigma_{c}^{-1} \mathbf{U}\right)^{-1} \mathbf{U}^{H} \Sigma_{c}^{-1} \mathbf{x}\right)}{F_{N}\left(\mathbf{x}^{H} \Sigma_{c}^{-1} \mathbf{x}\right)} \underset{<}{\underset{H_{0}}{>}} \eta
$$

For the specific case of Gaussian clutter, where $F_{N}(p)=e^{-p}$, Equation 17 can be further reduced to the following expression:

$$
\ln \frac{f_{x}\left(\mathbf{x} \mid H_{1}, \hat{\alpha}\right)}{f_{x}\left(\mathbf{x} \mid H_{0}\right)}=\mathbf{x}^{H} \Sigma_{c}^{-1} \mathbf{V}\left(\mathbf{V}^{H} \Sigma_{c}^{-1} \mathbf{V}\right)^{-1} \mathbf{V}^{H} \Sigma_{c}^{-1} \mathbf{x} \underset{H_{0}}{\stackrel{H_{1}}{>}} \ln \eta
$$

Explicit expressions for a trihedral and dihedral detector can be found by inserting the appropriate basis vector model into Equation 20. For the trihedral detector, $M=1$ and $\boldsymbol{V}=\left[\begin{array}{lll}1 & 0 & 1\end{array}\right]^{T} .{ }^{13,16}$ For the dihedral detector, $M=2, \mathbf{v}_{1}=\left[\begin{array}{lll}1 & 0 & -1\end{array}\right]^{\mathrm{T}}$, and $\mathbf{v}_{2}=\left[\begin{array}{ll}0 \\ 0\end{array}\right.$ $10]^{\mathrm{T}}$. This does not exactly match the Huynen model because each basis vector has an independent unknown phase. For the covariance model of Equation 3, the noncoherent dihedral detector for Gaussian clutter is the following:

$$
\begin{aligned}
& A|H H|^{2}-2 B \operatorname{Re}\left\{H H^{*} V V\right\}-2 C \operatorname{Im}\left\{H H^{*} V V\right\}+D|V V|^{2}+E|H V|^{2} \underset{H_{0}}{H_{1}} \ln \eta \\
& A=\frac{\gamma^{2}+|\rho|^{2} \gamma+2 \gamma \sqrt{\gamma} \operatorname{Re}\{\rho\}}{\left[\sigma_{H H} \gamma\left(1-|\rho|^{2}\right)\right](\gamma+1+2 \sqrt{\gamma} \operatorname{Re}\{\rho\})} \\
& B=\frac{\gamma+\gamma \operatorname{Re}\{\rho \rho\}+\sqrt{\gamma} \operatorname{Re}\{\rho\}+\gamma \sqrt{\gamma} \operatorname{Re}\{\rho\}}{\left[\sigma_{H H} \gamma\left(1-|\rho|^{2}\right)\right](\gamma+1+2 \sqrt{\gamma} \operatorname{Re}\{\rho\})} \\
& C=\frac{\gamma \operatorname{Im}\{\rho \rho\}+\sqrt{\gamma} \operatorname{Im}\{\rho\}+\gamma \sqrt{\gamma} \operatorname{Im}\{\rho\}}{\left[\sigma_{H H} \gamma\left(1-|\rho|^{2}\right)\right](\gamma+1+2 \sqrt{\gamma} \operatorname{Re}\{\rho\})} \\
& D=\frac{1+|\rho|^{2} \gamma+2 \gamma \sqrt{\gamma} \operatorname{Re}\{\rho\}}{\left[\sigma_{H H} \gamma\left(1-|\rho|^{2}\right)\right](\gamma+1+2 \sqrt{\gamma} \operatorname{Re}\{\rho\})} \\
& E=\frac{1}{\sigma_{H H} \varepsilon}
\end{aligned}
$$

We can simplify the noncoherent polarimetric subspace target detector by prewhitening the signal and basis vectors for the clutter statistics. The whitening transformation only changes the covariance and mean of the SIRV clutter statistics. ${ }^{10-12}$

$$
\begin{gathered}
\Sigma_{c} \Phi=\Phi \Lambda \\
\mathbf{x}_{w}=\Lambda^{-1 / 2} \Phi^{H} \mathbf{x} \\
\mathbf{V}_{w}=\Lambda^{-1 / 2} \Phi^{H} \mathbf{V} \\
\mathbf{U}_{w}=\Lambda^{-1 / 2} \Phi^{H} \mathbf{U}
\end{gathered}
$$

Equation 22 represents the eigendecomposition of the clutter covariance, $\Sigma_{c}$, where $\Phi$ contains the eigenvectors as column vectors and $\Lambda$ is a diagonal matrix containing the eigenvalues. The noncoherent polarimetric subspace target detector in terms of the whitened signal and basis vectors can be expressed as follows:

$$
\frac{f_{x}\left(\mathbf{x} \mid H_{1}, \hat{\alpha}\right)}{f_{x}\left(\mathbf{x} \mid H_{0}\right)}=\frac{F_{N}\left(\mathbf{x}_{w}^{H} \mathbf{U}_{w}\left(\mathbf{U}_{w}^{H} \mathbf{U}_{w}\right)^{-1} \mathbf{U}_{w}^{H} \mathbf{x}_{w}\right)}{F_{N}\left(\mathbf{x}_{w}^{H} \mathbf{x}_{w}\right)} \underset{H_{0}}{>}{ }_{<} \eta
$$


We can now interpret the structure of this detector in terms of projection operators in the whitened signal space. The numerator of Equation 24 is $F_{N}$ evaluated for the energy of the whitened signal that does not project onto the whitened signal basis vectors; the denominator of Equation 24 is $F_{N}$ evaluated for the energy of the whitened signal. Since $F_{N}$ is a strictly decreasing function, the likelihood ratio test produces high values when most of the whitened signal energy is contained in the subspace defined by the whitened basis vectors. In the special case of Gaussian clutter, the likelihood ratio test is just a measure of the energy of the whitened signal that projects onto the subspace defined by the whitened basis vectors. Figure 1 shows a representation of the clutter, dihedral signal space, sample signal, and the projection of the sample signal onto the dihedral signal space before and after a whitening transformation.
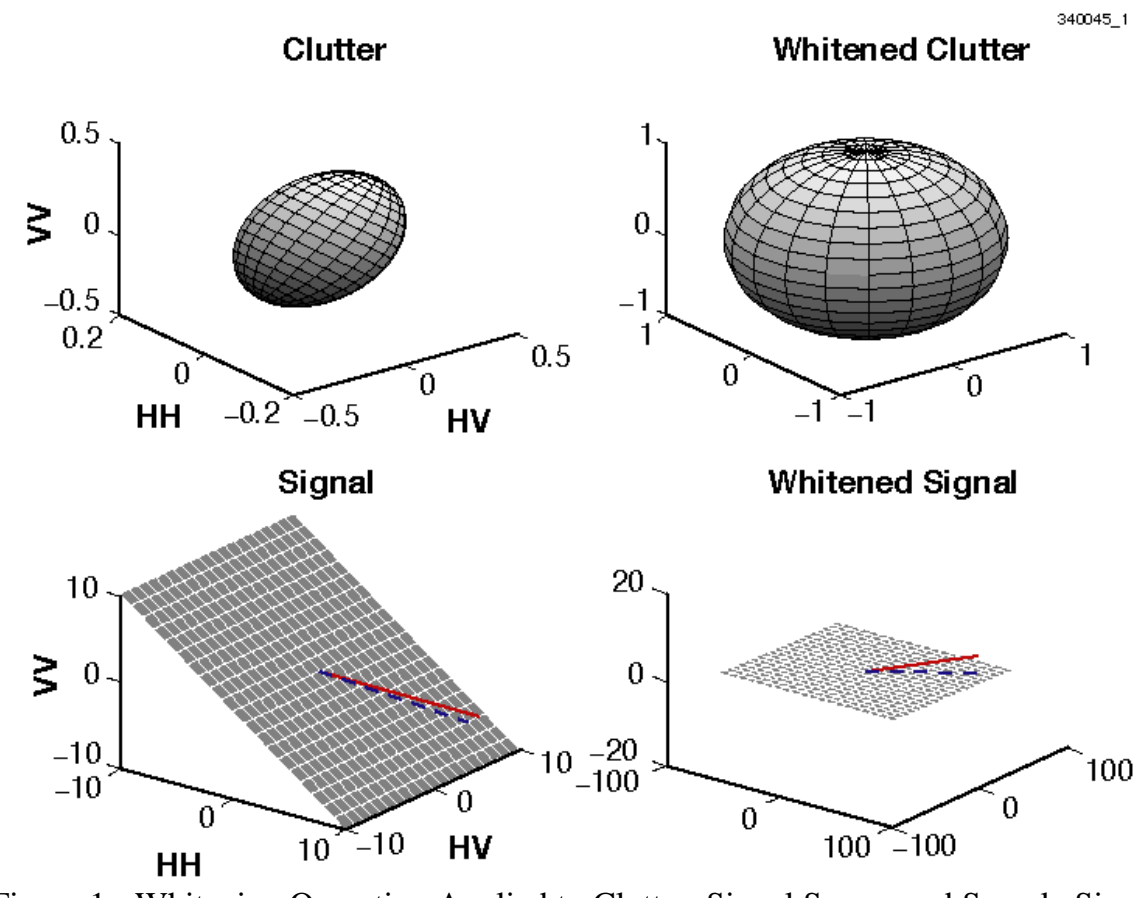

Figure 1 - Whitening Operation Applied to Clutter, Signal Space, and Sample Signal

When $\mathbf{V}_{w}$ spans the entire space (i.e. $M=N$ and the $\mathbf{v}_{i}$ are linearly independent), $\mathbf{V}_{w}\left(\mathbf{V}_{w}^{H} \mathbf{V}_{w}\right)^{-1} \mathbf{V}_{w}^{H}=\mathbf{I}, \boldsymbol{U}$ is the null space, the numerator of Equation 24 is $F_{N}(0)$, and the polarimetric subspace detector is exactly the PWF detector.

$$
\begin{aligned}
& \frac{f_{x}\left(\mathbf{x} \mid H_{1}, \hat{\alpha}\right)}{f_{x}\left(\mathbf{x} \mid H_{0}\right)}=\frac{F_{N}(0)}{F_{N}\left(\mathbf{x}_{w}^{H} \mathbf{x}_{w}\right)} \stackrel{H_{1}}{\underset{H_{0}}{>}} \eta \\
& \mathbf{x}_{w}^{H} \mathbf{x}_{w}=\mathbf{x}^{H} \Sigma_{c}^{-1} \mathbf{x}_{H_{0}}^{\stackrel{H_{1}}{<}} F_{N}^{-1}\left(\frac{F_{N}(0)}{\eta}\right)
\end{aligned}
$$

Note that the structure of the PWF detector is the same for any SIRV clutter model. The specific SIRV clutter model only effects the threshold of the test. This is not true for the polarimetric subspace target detector where the structure of the likelihood ratio test depends on the parameters of the SIRV clutter model.

\subsection{Derivation of coherent polarimetric subspace target detector}

The coherent polarimetric subspace target detector is now derived where the basis vectors representing the target signal have a common unknown phase. This version of the detector exactly matches the Huynen dihedral model for the scatterer. The GLRT can be expressed as follows: 


$$
\frac{\max _{\alpha_{R}, \phi} f_{x}\left(\mathbf{x} \mid H_{1}, \alpha_{R}, \phi\right)}{f_{x}\left(\mathbf{x} \mid H_{0}\right)}=\frac{\max _{\alpha_{R}, \phi} F_{N}\left(\left(\mathbf{x}-e^{j \phi} \mathbf{V} \alpha_{R}\right)^{H} \Sigma_{c}^{-1}\left(\mathbf{x}-e^{j \phi} \mathbf{V} \alpha_{R}\right)\right)}{F_{N}\left(\mathbf{x}^{H} \Sigma_{c}^{-1} \mathbf{x}\right)} \underset{<}{\underset{H_{0}}{<}} \eta
$$

where $F_{N}(p)$ is defined by Equation 12. For simplicity, Equation 26 is simplified to the clutter whitened space in the same manner as with the noncoherent polarimetric subspace target detector where the whitening transformation is defined by Equations 22 and 23 . Equation 26 can be equivalently expressed in terms of the whitened signal, $\mathbf{x}_{w}$, and whitened basis vectors, $\mathbf{V}_{w}$ :

$$
\frac{\max _{\alpha_{R}, \phi} f_{x}\left(\mathbf{x} \mid H_{1}, \alpha_{R}, \phi\right)}{f_{x}\left(\mathbf{x} \mid H_{0}\right)}=\frac{\max _{\alpha, \phi} F_{N}\left(\left(\mathbf{x}_{w}-e^{j \phi} \mathbf{V}_{w} \alpha_{R}\right)^{H}\left(\mathbf{x}_{w}-e^{j \phi} \mathbf{V}_{w} \alpha_{R}\right)\right)}{F_{N}\left(\mathbf{x}_{w}^{H} \mathbf{x}_{w}\right)} \underset{H_{0}}{>}{ }_{<}
$$

We maximize over $\alpha_{R}$ and $\phi$ just as with $\alpha$ for the noncoherent detector.

$$
\max _{\alpha_{R}, \phi} p=\left(\mathbf{x}_{w}-e^{j \phi} \mathbf{V}_{w} \alpha_{R}\right)^{H}\left(\mathbf{x}_{w}-e^{j \phi} \mathbf{V}_{w} \alpha_{R}\right)
$$

To simplify the maximization over $\alpha_{R}$ and $\phi$, we express $p$ in the following form:

$$
p=\mathbf{x}_{w}^{H} \mathbf{x}_{w}-2 \alpha_{R}^{T}\left[\cos \phi \operatorname{Re}\left\{\mathbf{V}_{w}^{H} \mathbf{x}_{w}\right\}+\sin \phi \operatorname{Im}\left\{\mathbf{V}_{w}^{H} \mathbf{x}_{w}\right\}\right]+\alpha_{R}^{T} \mathbf{V}_{w}^{H} \mathbf{V}_{w} \alpha_{R}
$$

We now take the derivative in terms of $\phi$ and $\alpha_{R}$ and set them equal to zero to find the maximum likelihood estimates of $\phi$ and $\alpha_{R}$ :

$$
\begin{gathered}
\frac{d p}{d \phi}=\left.\alpha_{R}^{T}\left[\sin \phi \operatorname{Re}\left\{\mathbf{V}_{w}^{H} \mathbf{x}_{w}\right\}-\cos \phi \operatorname{Im}\left\{\mathbf{V}_{w}^{H} \mathbf{x}_{w}\right\}\right]\right|_{\phi=\hat{\phi}}=0 \\
\frac{\partial p}{\partial \alpha_{R}}=-2\left[\cos \phi \operatorname{Re}\left\{\mathbf{V}_{w}^{H} \mathbf{x}_{w}\right\}+\sin \phi \operatorname{Im}\left\{\mathbf{V}_{w}^{H} \mathbf{x}_{w}\right\}\right]+\left.2 \mathbf{V}_{w}^{H} \mathbf{V}_{w} \alpha_{R}\right|_{\alpha_{R}=\hat{\alpha}_{R}}=\mathbf{0} \\
\hat{\alpha}_{R}=\left(\mathbf{V}_{w}^{H} \mathbf{V}_{w}\right)^{-1}\left[\cos \phi \operatorname{Re}\left\{\mathbf{V}_{w}^{H} \mathbf{x}_{w}\right\}+\sin \phi \operatorname{Im}\left\{\mathbf{V}_{w}^{H} \mathbf{x}_{w}\right\}\right]
\end{gathered}
$$

The following definitions are used to simplify the equations:

$$
\begin{gathered}
\mathbf{z}_{r} \stackrel{\Delta}{=} \operatorname{Re}\left\{\mathbf{V}_{w}^{H} \mathbf{x}_{w}\right\} \\
\stackrel{\Delta}{\mathbf{z}_{i}}=\operatorname{Im}\left\{\mathbf{V}_{w}^{H} \mathbf{x}_{w}\right\} \\
\mathbf{A} \stackrel{\Delta}{=}\left(\mathbf{V}_{w}^{H} \mathbf{V}_{w}\right)^{-1}
\end{gathered}
$$

Equations 30 and 31 are now combined to solve for the maximum likelihood estimate of the phase, $\phi$ :

$$
\left[\cos \hat{\phi} \mathbf{z}_{r}^{T}+\sin \hat{\phi} \mathbf{z}_{i}^{T}\right] \mathbf{A}\left[\sin \hat{\phi} \mathbf{z}_{r}-\cos \hat{\phi} \mathbf{z}_{i}\right]=0
$$

This expression can be simplified using double angle trigonometric relationships:

$$
\begin{aligned}
& \frac{1}{2} \sin (2 \phi)\left(\mathbf{z}_{r}^{T} \mathbf{A} \mathbf{z}_{r}-\mathbf{z}_{i}^{T} \mathbf{A} \mathbf{z}_{i}\right)-\cos (2 \phi) \mathbf{z}_{r}^{T} \mathbf{A} \mathbf{z}_{i}=0 \\
& \hat{\phi}=\frac{1}{2} \tan ^{-1}\left(\frac{\mathbf{z}_{r}^{T} \mathbf{A} \mathbf{z}_{i}}{\frac{1}{2}\left[\mathbf{z}_{r}^{T} \mathbf{A} \mathbf{z}_{r}-\mathbf{z}_{i}^{T} \mathbf{A} \mathbf{z}_{i}\right]}\right)
\end{aligned}
$$


Equation 34 expresses the maximum likelihood estimate of the phase. The maximum likelihood estimate of $\alpha_{R}$ is found by inserting Equation 34 into Equation 31. The GLRT of Equation 27 can now be expressed in terms of the maximum likelihood estimates of Equations 34 and 31:

$$
\begin{aligned}
& \frac{\max _{\alpha_{R}, \phi} f_{x}\left(\mathbf{x} \mid H_{1}, \alpha_{R}, \phi\right)}{f_{x}\left(\mathbf{x} \mid H_{0}\right)}=\frac{F_{N}\left(\mathbf{x}_{w}^{H} \mathbf{x}_{w}-2 \hat{\alpha}_{R}^{T}\left[\cos \hat{\phi} \mathbf{z}_{r}+\sin \hat{\phi} \mathbf{z}_{i}\right]+\hat{\alpha}_{R}^{T} \mathbf{V}_{w}^{H} \mathbf{V}_{w} \hat{\alpha}_{R}\right)}{F_{N}\left(\mathbf{x}_{w}^{H} \mathbf{x}_{w}\right)} \underset{H_{0}}{\stackrel{H_{1}}{>}} \eta \\
& =\frac{F_{N}\left(\mathbf{x}_{w}^{H} \mathbf{x}_{w}-\frac{1}{2}\left[\mathbf{z}_{r}^{T} \mathbf{A} \mathbf{z}_{r}+\mathbf{z}_{i}^{T} \mathbf{A} \mathbf{z}_{i}\right]-\frac{1}{2} \cos (2 \hat{\phi})\left[\mathbf{z}_{r}^{T} \mathbf{A} \mathbf{z}_{r}-\mathbf{z}_{i}^{T} \mathbf{A} \mathbf{z}_{i}\right]-\sin (2 \hat{\phi}) \mathbf{z}_{r}^{T} \mathbf{A} \mathbf{z}_{i}\right)}{F_{N}\left(\mathbf{x}_{w}^{H} \mathbf{x}_{w}\right)} \underset{H_{0}}{>}{ }_{i} \eta \\
& =\frac{F_{N}\left(\mathbf{x}_{w}^{H} \mathbf{x}_{w}-\frac{1}{2}\left[\mathbf{z}_{r}^{T} \mathbf{A} \mathbf{z}_{r}+\mathbf{z}_{i}^{T} \mathbf{A} \mathbf{z}_{i}\right]-\sqrt{\frac{1}{4}\left(\mathbf{z}_{r}^{T} \mathbf{A} \mathbf{z}_{r}-\mathbf{z}_{i}^{T} \mathbf{A} \mathbf{z}_{i}\right)^{2}+\left(\mathbf{z}_{r}^{T} \mathbf{A} \mathbf{z}_{i}\right)^{2}}\right)}{F_{N}\left(\mathbf{x}_{w}^{H} \mathbf{x}_{w}\right)} \underset{H_{0}}{>} H_{1} \eta
\end{aligned}
$$

Double angle trigonometric relationships are again used to simplify the expression. We can express $\cos 2 \phi$ and $\sin 2 \phi$ in terms of the numerator and denominator of $\tan 2 \phi$. Just as with the noncoherent detector the general SIRV detector can be simplified to the case of Gaussian clutter:

$$
\ln \frac{f_{x}\left(\mathbf{x} \mid H_{1}, \hat{\alpha}_{R}, \hat{\phi}\right)}{f_{x}\left(\mathbf{x} \mid H_{0}\right)}=\frac{1}{2}\left[\mathbf{z}_{r}^{T} \mathbf{A} \mathbf{z}_{r}+\mathbf{z}_{i}^{T} \mathbf{A} \mathbf{z}_{i}\right]+\sqrt{\frac{1}{4}\left(\mathbf{z}_{r}^{T} \mathbf{A} \mathbf{z}_{r}-\mathbf{z}_{i}^{T} \mathbf{A} \mathbf{z}_{i}\right)^{2}+\left(\mathbf{z}_{r}^{T} \mathbf{A} \mathbf{z}_{i}\right)^{2}} \underset{H_{0}}{\stackrel{H_{1}}{>} \ln \eta}
$$

The specific forms of this detector for the trihedral and dihedral are found by inserting the appropriate values for $\mathbf{V}$, just as with the noncoherent detector.

\section{ADAPTIVE POLARIMETRIC SUBSPACE TARGET DETECTOR}

In this Section constant false alarm rate (CFAR) extensions to the detectors of Section 2 are developed for the special case of Gaussian clutter. It is straightforward to find CFAR versions of the noncoherent and coherent polarimetric subspace target detectors when the clutter is Gaussian because maximum likelihood estimates of the clutter parameters are available. The formulation of a CFAR detector for non-Gaussian clutter is left for future research primarily because it is not clear that it is possible to find closed form maximum likelihood estimates of the parameters of the SIRV distribution when the data is nonGaussian and more than one clutter sample is available. ${ }^{24-26}$ We use a well established approach employed by many others to find CFAR detectors for targets in Gaussian clutter using the GLRT approach and a set of auxiliary clutter samples. ${ }^{7,8,18-21}$ For our SAR target detection problem, the auxiliary clutter samples are drawn from a square template twice as large as the largest target that surrounds a center test pixel. ${ }^{5}$ We derive two sets of CFAR detectors. The first are for the case where the clutter statistics are completely known except for an amplitude factor in the covariance. This is useful because this amplitude covariance factor varies more than any other parameter. The second set of CFAR detectors we present are for the case where the clutter covariance is completely unknown.

\subsection{Known Clutter Covariance Structure With Unknown Amplitude Factor}

We now derive CFAR versions of the noncoherent and coherent polarimetric subspace target detectors when the clutter is Gaussian and its covariance has an unknown amplitude factor. We have $K$ auxiliary independent clutter only samples available and a single test sample that may contain the target scatterer:

$$
\begin{array}{llc}
H_{1}: \mathbf{x}=\mathbf{x}_{s}(\theta)+\sqrt{a} \mathbf{x}_{c} & \mathbf{x}_{i}=\sqrt{a} \mathbf{x}_{c} & i=1,2, \ldots, K \\
H_{0}: \mathbf{x}=\sqrt{a} \mathbf{x}_{c} & \mathbf{x}_{i}=\sqrt{a} \mathbf{x}_{c} & \mathbf{x}_{c} \sim C N\left(\mathbf{0}, \Sigma_{c}\right)
\end{array}
$$

where $\theta$ represents the unknown parameters of the signal for either the noncoherent or coherent signal models. The $K$ auxiliary samples are independent from each other and from the test sample. We can express the pdfs of each hypothesis in terms of the unknown parameters: 


$$
\begin{aligned}
& f\left(\mathbf{x}, \mathbf{x}_{1}, \ldots, \mathbf{x}_{K} \mid H_{1}, a, \theta\right)=\left[\frac{1}{\pi^{N} a^{N}\left|\Sigma_{c}\right|}\right]^{K+1} \exp \left[-\frac{\left(\mathbf{x}-\mathbf{x}_{s}(\theta)\right)^{H} \Sigma_{c}^{-1}\left(\mathbf{x}-\mathbf{x}_{s}(\theta)\right)+\sum_{k=1}^{K} \mathbf{x}_{k}^{H} \Sigma_{c}^{-1} \mathbf{x}_{k}}{a}\right] \\
& f\left(\mathbf{x}, \mathbf{x}_{1}, \ldots, \mathbf{x}_{K} \mid H_{0}, a\right)=\left[\frac{1}{\pi^{N} a^{N}\left|\Sigma_{c}\right|}\right]^{K+1} \exp \left[-\frac{\mathbf{x}^{H} \Sigma_{c}^{-1} \mathbf{x}+\sum_{k=1}^{K} \mathbf{x}_{k}^{H} \Sigma_{c}^{-1} \mathbf{x}_{k}}{a}\right]
\end{aligned}
$$

The GLRT is again used to solve this problem where each hypothesis is maximized over its unknown nonrandom parameters:

$$
\lambda\left(\mathbf{x}, \mathbf{x}_{1}, \ldots, \mathbf{x}_{K}\right)=\frac{\max _{\alpha, a} f\left(\mathbf{x}, \mathbf{x}_{1}, \ldots, \mathbf{x}_{K} \mid H_{1}, \theta, a\right)}{\max _{a} f\left(\mathbf{x}, \mathbf{x}_{1}, \ldots, \mathbf{x}_{K} \mid H_{0}, a\right)}=\max _{\theta} \frac{f\left(\mathbf{x}, \mathbf{x}_{1}, \ldots, \mathbf{x}_{K} \mid H_{1}, \theta, \hat{a}\right)}{f\left(\mathbf{x}, \mathbf{x}_{1}, \ldots, \mathbf{x}_{K} \mid H_{0}, \hat{a}\right)} \underset{H_{0}}{>} \lambda_{0}
$$

The maximum likelihood estimates of the unknown amplitude, $a$, for each hypothesis are the following:

$$
\begin{aligned}
& \text { under } H_{1}: \quad \hat{a}=\frac{1}{(K+1) N}\left[\left(\mathbf{x}-\mathbf{x}_{s}(\theta)\right)^{H} \Sigma_{c}^{-1}\left(\mathbf{x}-\mathbf{x}_{s}(\theta)\right)+\sum_{k=1}^{K} \mathbf{x}_{k}^{H} \Sigma_{c}^{-1} \mathbf{x}_{k}\right] \\
& \text { under } H_{0}: \quad \hat{a}=\frac{1}{(K+1) N}\left[\mathbf{x}^{H} \Sigma_{c}^{-1} \mathbf{x}+\sum_{k=1}^{K} \mathbf{x}_{k}^{H} \Sigma_{c}^{-1} \mathbf{x}_{k}\right]
\end{aligned}
$$

The GLRT is now expressed in terms of these maximum likelihood estimates for $a$ :

$$
\lambda\left(\mathbf{x}, \mathbf{x}_{1}, \ldots, \mathbf{x}_{K}\right)=\max _{\theta}\left[\frac{\mathbf{x}^{H} \Sigma_{c}^{-1} \mathbf{x}+\sum_{k=1}^{K} \mathbf{x}_{k}^{H} \Sigma_{c}^{-1} \mathbf{x}_{k}}{\left(\mathbf{x}-\mathbf{x}_{s}(\theta)\right)^{H} \Sigma_{c}^{-1}\left(\mathbf{x}-\mathbf{x}_{s}(\theta)\right)+\sum_{k=1}^{K} \mathbf{x}_{k}^{H} \Sigma_{c}^{-1} \mathbf{x}_{k}}\right]^{(K+1) N} \begin{aligned}
& H_{1} \\
& \underset{<}{>} \lambda_{0} \\
& H_{0}
\end{aligned}
$$

We can maximize over $\theta$ by minimizing the quadratic in the denominator over $\theta$.

$$
\min _{\theta} p=\min _{\theta}\left(\mathbf{x}-\mathbf{x}_{s}(\theta)\right)^{H} \Sigma_{c}^{-1}\left(\mathbf{x}-\mathbf{x}_{s}(\theta)\right)
$$

In Section 2, for the case of Gaussian clutter, we solved for $\max _{\theta} e^{-p}$ which is equivalent to $\min _{\theta} p$, so the parameter estimates of Section 2 can be used to complete these CFAR detector derivations. The CFAR version of the noncoherent polarimetric subspace target detector is:

$$
\lambda_{1}\left(\mathbf{x}, \mathbf{x}_{1}, \ldots, \mathbf{x}_{K}\right)=\frac{\mathbf{x}^{H} \Sigma_{c}^{-1} \mathbf{x}+\sum_{k=1}^{K} \mathbf{x}_{k}^{H} \Sigma_{c}^{-1} \mathbf{x}_{k}}{\mathbf{x}^{H} \Sigma_{c}^{-1} \mathbf{x}-\mathbf{x}^{H} \Sigma_{c}^{-1} \mathbf{V}\left(\mathbf{V}^{H} \Sigma_{c}^{-1} \mathbf{V}\right)^{-1} \mathbf{V}^{H} \Sigma_{c}^{-1} \mathbf{x}+\sum_{k=1}^{K} \mathbf{x}_{k}^{H} \Sigma_{c}^{-1} \mathbf{x}_{k}} \stackrel{H_{1}}{\underset{H}{<}} \lambda_{0}
$$

The CFAR version of the coherent polarimetric subspace target detector is:

$$
\begin{gathered}
\lambda_{2}\left(\mathbf{x}, \mathbf{x}_{1}, \ldots, \mathbf{x}_{K}\right)= \\
\mathbf{x}^{H} \Sigma_{c}^{-1} \mathbf{x}+\sum_{k=1}^{K} \mathbf{x}_{k}^{H} \Sigma_{c}^{-1} \mathbf{x}_{k} \\
\mathbf{x}^{H} \Sigma_{c}^{-1} \mathbf{x}-\frac{1}{2}\left[\mathbf{z}_{r}^{T} \mathbf{A} \mathbf{z}_{r}+\mathbf{z}_{i}^{T} \mathbf{A} \mathbf{z}_{i}\right]-\sqrt{\frac{1}{4}\left(\mathbf{z}_{r}^{T} \mathbf{A} \mathbf{z}_{r}-\mathbf{z}_{i}^{T} \mathbf{A} \mathbf{z}_{i}\right)^{2}+\left(\mathbf{z}_{r}^{T} \mathbf{A} \mathbf{z}_{i}\right)^{2}+\sum_{k=1}^{K} \mathbf{x}_{k}^{H} \Sigma_{c}^{-1} \mathbf{x}_{k}} \\
H_{0} \\
H_{0}
\end{gathered} \lambda_{1}
$$

where $\mathbf{z}$ and $\mathbf{A}$ are defined by Equation 32. The noncoherent polarimetric subspace detector of Equation 43 can be represented in a very simple form by making use of the whitened subspace orthogonal to the whitened target subspace defined in Equation 18: 


$$
\lambda_{1}\left(\mathbf{x}, \mathbf{x}_{1}, \ldots, \mathbf{x}_{K}\right)=\frac{\mathbf{x}^{H} \Sigma_{c}^{-1} \mathbf{V}\left(\mathbf{V}^{H} \Sigma_{c}^{-1} \mathbf{V}\right)^{-1} \mathbf{V}^{H} \Sigma_{c}^{-1} \mathbf{x}}{\mathbf{x}^{H} \Sigma_{c}^{-1} \mathbf{U}\left(\mathbf{U}^{H} \Sigma_{c}^{-1} \mathbf{U}\right)^{-1} \mathbf{U}^{H} \Sigma_{c}^{-1} \mathbf{x}+\sum_{k=1}^{K} \mathbf{x}_{k}^{H} \Sigma_{c}^{-1} \mathbf{x}_{k}} \underset{H_{0}}{H_{1}} \lambda_{1}-1
$$

This form of the adaptive noncoherent polarimetric subspace detector has a very intuitive form. We find the energy of the whitened signal in the whitened target subspace based on the known structure of the clutter covariance. The output of this nonadaptive form of the detector contains the factor $a$, so we divide by an estimate of $a$ to remove this unknown parameter. The estimate of $a$ uses all available data including both the auxiliary samples and the subspace orthogonal to the target subspace. In the real SAR target detection problem, some target energy will not be in the dihedral subspace and se we do not include the first term of the denominator. In our application, $K$ is large, so we don't lose any performance by not using the first denominator term.

\subsection{Clutter Covariance Completely Unknown}

We now present CFAR versions of the noncoherent and coherent polarimetric subspace target detectors when the clutter is Gaussian and its covariance is completely unknown. We again have $K$ auxiliary clutter only samples available to estimate the clutter statistics and a single test sample that may contain the target scatterer:

$$
\begin{array}{llc}
H_{1}: \mathbf{x}=\mathbf{x}_{s}(\theta)+\mathbf{x}_{c} & \mathbf{x}_{i}=\mathbf{x}_{c} & i=1,2, \ldots, K \\
H_{0}: \mathbf{x}=\mathbf{x}_{c} & \mathbf{x}_{i}=\mathbf{x}_{c} & \mathbf{x}_{c} \sim C N\left(\mathbf{0}, \Sigma_{c}\right)
\end{array}
$$

where $\theta$ represents the unknown parameters of the signal for either the noncoherent or coherent signal models and $\Sigma_{c}$ now represents a completely unknown covariance. The $K$ auxiliary samples are again independent from each other and from the test sample. The derivation of the CFAR detector with unknown covariance follows directly from the work of Kelly. ${ }^{18,19}$ In fact the adaptive noncoherent dihedral detector is a special case of the Kelly-Forsythe detector. ${ }^{19}$ The noncoherent polarimetric subspace CFAR target detector for unknown covariance is:

$$
\frac{1+\mathbf{x}^{H} \hat{\Sigma}_{c}^{-1} \mathbf{x}}{1+\mathbf{x}^{H} \hat{\Sigma}_{c}^{-1} \mathbf{x}-\mathbf{x}^{H} \hat{\Sigma}_{c}^{-1} \mathbf{V}\left(\mathbf{V}^{H} \hat{\Sigma}_{c}^{-1} \mathbf{V}\right)^{-1} \mathbf{V}^{H} \hat{\Sigma}_{c}^{-1} \mathbf{x}} \stackrel{H_{1}}{\underset{H_{0}}{>} \lambda_{2}}
$$

The coherent polarimetric subspace CFAR target detector for unknown covariance is:

$$
\frac{1+\mathbf{x}^{H} \hat{\Sigma}_{c}^{-1} \mathbf{x}}{1+\mathbf{x}^{H} \hat{\Sigma}_{c}^{-1} \mathbf{x}-\frac{1}{2}\left[\mathbf{z}_{r}^{T} \mathbf{A} \mathbf{z}_{r}+\mathbf{z}_{i}^{T} \mathbf{A} \mathbf{z}_{i}\right]-\sqrt{\frac{1}{4}\left(\mathbf{z}_{r}^{T} \mathbf{A} \mathbf{z}_{r}-\mathbf{z}_{i}^{T} \mathbf{A} \mathbf{z}_{i}\right)^{2}+\left(\mathbf{z}_{r}^{T} \mathbf{A} \mathbf{z}_{i}\right)^{2}}} \stackrel{H_{1}}{\underset{<}{H_{0}}} \lambda_{2}
$$

where,

$$
\begin{aligned}
& \mathbf{z}_{r} \stackrel{\Delta}{=} \operatorname{Re}\left\{\mathbf{V}^{H} \hat{\Sigma}_{c}^{-1} \mathbf{x}\right\} \\
& \stackrel{\Delta}{=} \operatorname{Im}\left\{\mathbf{V}^{H} \hat{\Sigma}_{c}^{-1} \mathbf{x}\right\} \\
& \mathbf{A} \stackrel{\Delta}{=}\left(\mathbf{V}^{H} \hat{\Sigma}_{c}^{-1} \mathbf{V}\right)^{-1}
\end{aligned}
$$

\section{DETECTION PERFORMANCE}

In this Section performance results are presented for the detectors developed in Section 2 and 3. The performance results are developed based on real polarimetric SAR data. The polarimetric SAR data used to evaluate these detectors was collected by the MIT Lincoln Laboratory using the Advanced Detection Technology Sensor (ADTS). ${ }^{27}$ This sensor collects fully polarimetric one foot resolution SAR data at a nominal operating frequency of $33 \mathrm{Ghz}$. We have also developed analytical performance expressions for the noncoherent polarimetric subspace detector. ${ }^{26}$ The experiments on real SAR data do a better job of predicting performance than the analytical expressions. The analytical performance expressions are, however, useful because they show how the parameters of the target and clutter statistics effect performance. 
In Section 4.1 clutter parameter estimates are found. Estimates of the clutter parameters are required to implement the CFAR detector with known covariance structure developed in Section 3 and to understand the Gaussianity of the data. In Section 4.2 performance results are presented for both the noncoherent and coherent detectors against real millimeter-wave polarimetric SAR data.

\subsection{Estimation of Clutter Parameters}

In this Section, estimates of the clutter parameters are presented. The clutter data is not homogeneous, so separate estimates are found of the clutter covariance and the Gamma parameter $(v)$ for different homogeneous regions of clutter. We show the empirical marginal distributions of the clutter versus the marginal distributions predicted by the Gaussian and $K$-distribution models to evaluate the quality of the $K$-distribution fit to the clutter data.

The clutter data estimates in this section are based on three homogeneous regions of clutter and a fourth region containing a mixture of the different kinds of clutter. The first SAR image used to estimate the clutter parameters is a $512 \mathrm{x} 512$ pixel region of grass clutter. The second SAR image is a 512 x 512 pixel region of tree clutter. The third SAR image is a $128 \times 128$ pixel region of water clutter. The fourth SAR image is a 512 x 512 pixel region containing grass, tree, and water clutter. The $128 \mathrm{x}$ 128 pixel region of water clutter is drawn from a small pond contained in the $512 \times 512$ pixel mixed image.

Table 1 shows estimates of the covariance parameters based on the grass, tree, water, and mixed clutter data sets. These estimates were found using the sample covariance estimator. ${ }^{38}$

Table 1 - Polarimetric Covariance Parameters of Clutter Data

\begin{tabular}{|l|l|l|l|l|}
\hline & $\sigma_{H H}$ & $\varepsilon$ & $\gamma$ & $\rho$ \\
\hline Grass & 0.0546 & 0.1801 & 1.005 & $0.5047+\mathrm{j} 0.05891$ \\
\hline Trees & 0.1147 & 0.1143 & 1.024 & $0.7620+\mathrm{j} 0.01432$ \\
\hline Mixed & 0.0713 & 0.1599 & 1.021 & $0.5554+\mathrm{j} 0.04707$ \\
\hline Water & 0.0041 & 0.3026 & 1.232 & $0.4885+\mathrm{j} 0.05694$ \\
\hline
\end{tabular}

Once we have estimates of the covariance, we must estimate the scalar parameter of the SIRV $K$-distribution. This parameter estimate is based on the polarimetric SAR data and the estimate of the covariance. When the data approaches the Gaussian distribution the scalar parameter of the SIRV $K$-distribution approaches infinity. We use the following estimator for the scalar parameter of the SIRV K-distributed data: ${ }^{38}$

$$
\hat{v}=\frac{12}{\frac{1}{K} \sum_{i=1}^{K}\left(\mathbf{x}_{i}^{H} \hat{\Sigma}_{c}^{-1} \mathbf{x}_{i}\right)_{i}^{2}-12}
$$

The estimate of $v$ for the grass, tree, and mixed clutter are shown in Table 2. We did not compute the estimate for the water clutter because we only had a small number of samples. Note that the grass data has a relatively high value of $v$ indicating that it is more Gaussian.

Table 2 - $K$-Distribution Parameter of Polarimetric Clutter Data

\begin{tabular}{|l|l|}
\hline & $v$ \\
\hline Grass & 5.7173 \\
\hline Trees & 0.7912 \\
\hline Mixed & 0.6297 \\
\hline
\end{tabular}




\subsection{Detection Performance}

In this Section we present the performance of our dihedral detectors against real millimeter-wave polarimetric SAR data. We evaluated the detectors against $3.75 \mathrm{~km}^{2}$ of clutter data that includes a mix of grass, trees, and manmade cultural clutter. We evaluated the detection performance against two target sets: (1) 84 bare tactical targets and (2) 79 camouflaged tactical targets. We have tested three different versions of the detector: (1) the adaptive CFAR test of Equation 45, (2) the nonadaptive test of Equation 20 followed by the two parameter CFAR test, ${ }^{5}$ and (3) the nonadaptive test of Equation 36 followed by the two parameter CFAR test. ${ }^{5}$ In all cases we implement the detectors with the mixed clutter parameters of Table 1 . We implemented the CFAR detectors with a 61 x 61 CFAR stencil. The detector output for each test is followed by a simple clustering algorithm so that only one detection or false alarm is reported in a given target sized area. ${ }^{5}$ We evaluated the performance of these detectors with two signal models: (1) completely unknown signal (i.e., the PWF) and (2) the dihedral signal model. Figures 2-5 show ROC curves depicting the performance of these detectors against bare and camouflaged targets.

\section{CONCLUSIONS AND FUTURE WORK}

We have developed a new dihedral detector for polarimetric SAR data based on a subspace model. For the case of known clutter parameters, we have developed a test for the dihedral signal embedded in additive SIRV clutter including the $K$ distribution. We have shown that the structure of the PWF detector is that same for any SIRV clutter model, demonstrating theoretically why the PWF detector has had very robust performance against real SAR data. For the case of unknown clutter parameters, we have developed a test for the dihedral signal embedded in additive Gaussian clutter. We have demonstrated the performance of this new dihedral detector using real millimeter-wave polarimetric SAR data and have shown that it performs better than the PWF detector which assumes that the structure of the polarimetric target signal is completely unknown. We are currently evaluating whether the nonadaptive, non-Gaussian dihedral detector will lead to any performance improvement against the real SAR data which has been shown to better fit the $K$-distribution.

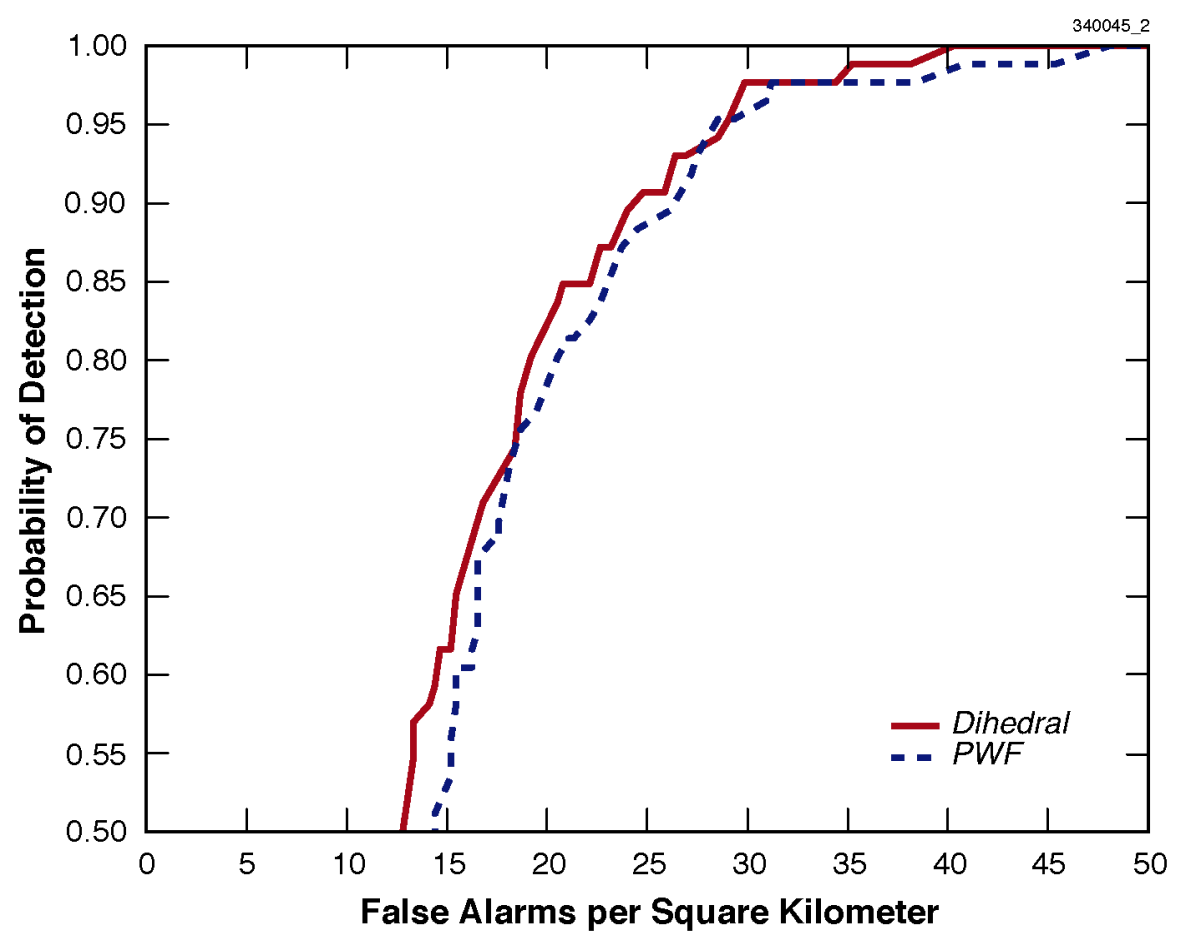

Figure 2 - Adaptive CFAR Detector Against Bare Tactical Targets 


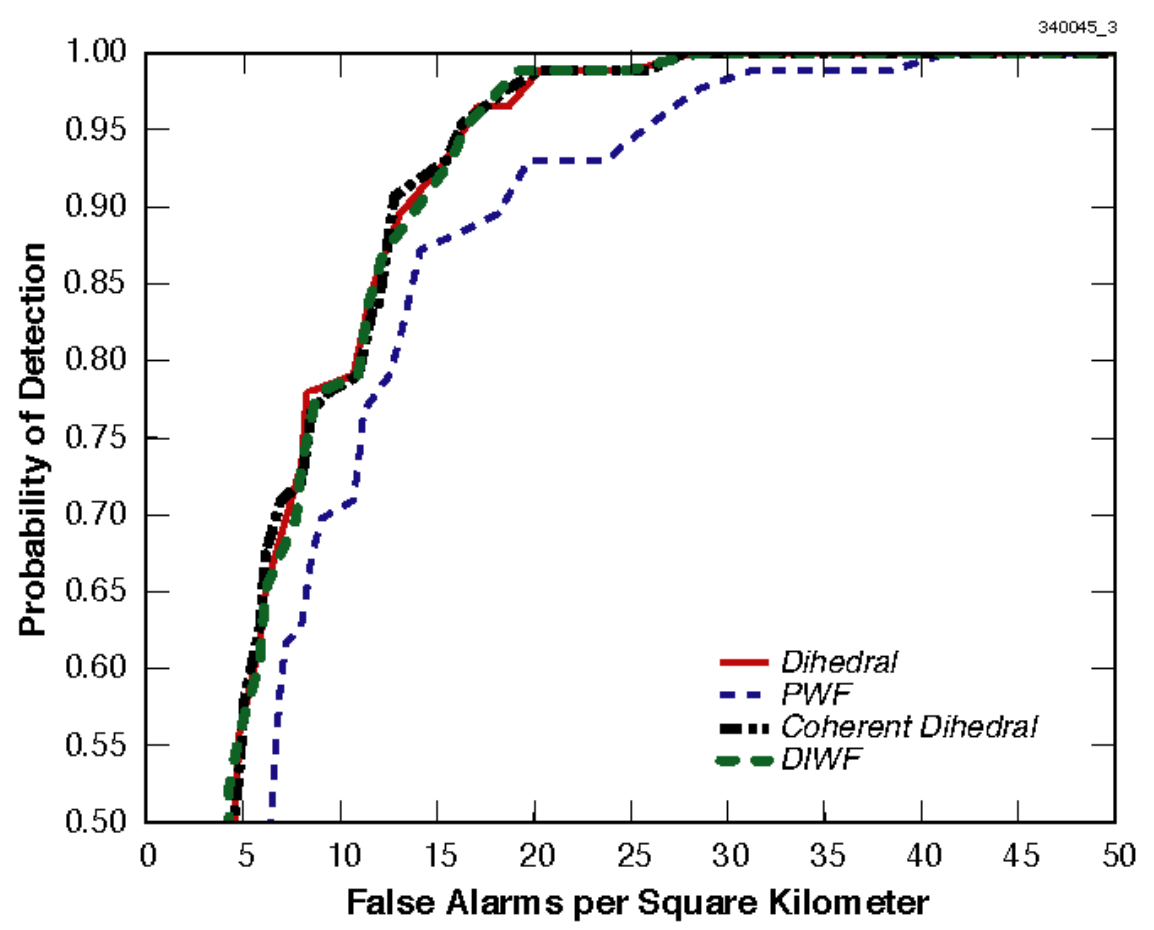

Figure 3 - Adaptive CFAR Detector Against Camouflaged Tactical Targets

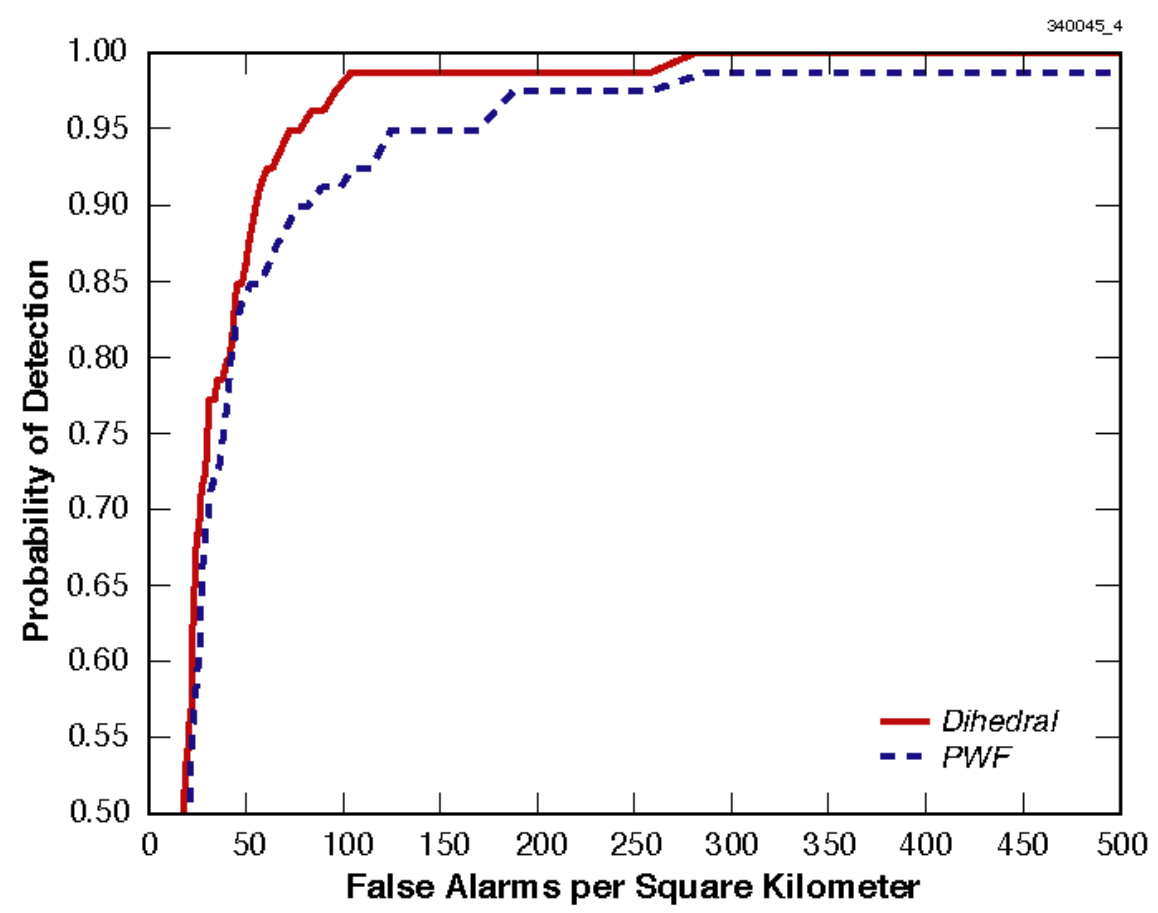

Figure 4 - Nonadaptive Detector Followed by 2-Parameter CFAR Against Bare Tactical Targets 


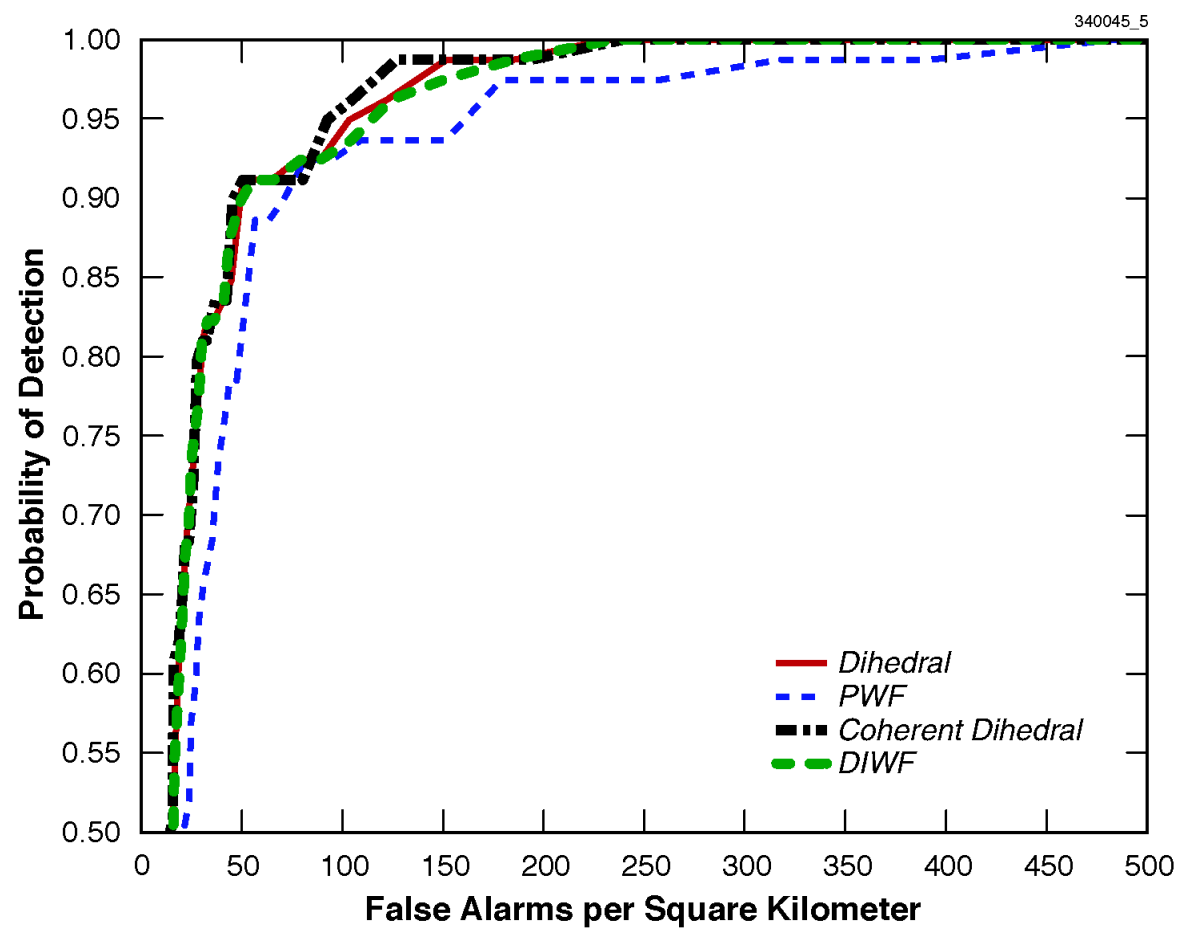

Figure 5 - Nonadaptive Detector Followed by 2-Parameter CFAR Against Camouflaged Tactical Targets

\section{APPENDIX}

The dihedral whitening filter (DIWF) detector developed by Michael B. Sechtin is just the PWF combination of the two channels which respond best to a dihedral (the $H V$ and $H H$ - $V V$ channels). ${ }^{17}$ The DIWF can be expressed as follows:

$$
\mathbf{x}^{H} \mathbf{V}\left(\mathbf{V}^{H} \Sigma_{c} \mathbf{V}\right)^{-1} \mathbf{V}^{H} \mathbf{x} \underset{H_{0}}{\stackrel{H_{1}}{>}} \lambda_{3}
$$

where $\boldsymbol{V}$ is defined just as with the dihedral detectors developed in this paper. When the clutter covariance has the structure of Equation 3. The DIWF detector can be expressed as follows:

$$
\frac{|H H-V V|^{2}}{\sigma_{H H}(1+\gamma-2 \sqrt{\gamma} \operatorname{Re}\{\rho\})}+\frac{|H V|^{2}}{\sigma_{H H} \varepsilon} \underset{H_{0}}{>}{ }_{i} \lambda_{3}
$$

\section{REFERENCES}

1. D. E. Kreithen, S. D. Halverson, and G. J. Owirka, "Discriminating Targets from Clutter," MIT Lincoln Laboratory Journal, Vol. 6, No. 1, 1993, pp. 25-52.

2. L. Novak, G. Owirka, and C. Netishen, "Performance of a High-Resolution SAR Automatic Target Recognition System," MIT Lincoln Laboratory Journal, Vol. 6, No. 1, 1993, pp. 11-24.

3. L. Novak, M. Sechtin, and M. Cardullo, "Studies of Target Detection Algorithms That Use Polarimetric Radar Data," IEEE Trans. on AES, Vol. 25, No. 2, March 1989, pp. 150-165.

4. L. Novak and M. Burl, "Optimal Speckle Reduction in Polarimetric SAR Imagery," IEEE Trans. on AES, Vol. 26, No. 2, March 1990, pp. 293-305. 
5. L. Novak, M. Burl, and W. Irving, "Optimal Polarimetric Processing for Enhanced Target Detection," IEEE Trans. on AES, Vol. 29, No. 1, January 1993, pp. 234-244.

6. L. Novak and S. Hesse, "Optimal Polarizations for Radar Detection and Recognition of Targets in Clutter," IEEE National Radar Conference, 1993.

7. H. Wang and C. Lee, "Adaptive Array Processing for Real-Time Airborne Radar Detection of Critical Mobile Targets," Adaptive Antenna Systems Symposium, Long Island, NY, 1992.

8. H. Park, C. Lee, and H. Wang, "A Novel Adaptive Polarimetric Processor With Embedded CFAR," Submitted to IEEE AES, January 1994.

9. J.R. Huynen, Phenomenological Theory of Radar Targets. Ph.D. Thesis, Technical University, Delft, The Netherlands, 1970.

10. E. Conte and M. Longo, "Characterisation of radar clutter as a spherically invariant random process," IEE Proceedings, Part F, Communications, Radar \& Signal Processing, Vol. 134, No. 2, April 1987, pp. 191-197.

11. M. Rangaswamy, D. Weiner, and A. Ozturk, "Non-Gaussian Random Vector Identification Using Spherically Invariant Random Processes," IEEE Trans. on AES, Vol. 29, No. 1, Jan. 1993, pp. 111-124.

12. K. Sangston and K. Gerlach, "Coherent Detection of Radar Targets in a Non-Gaussian Background," IEEE Trans. on AES, Vol. 30, No. 2, April 1994, pp. 330-340.

13. S. R. De Graaf, "SAR Image Enhancement via Adaptive Polarization Synthesis and Polarimetric Detection Performance," Presented at the Polarimetric Technology Workshop, GACIAC 88-03, 16-18 August, 1988.

14. W. L. Cameron and L. K. Leung, "Feature Motivated Polarization Scattering Matrix Decomposition," Proceedings of the 1990 IEEE International Radar Conference, 1990, pp. 549-557.

15. R. Dilsavor, Detection of Target Scattering Centers in Terrain Clutter Using an Ultra-Wideband, Fully-Polarimetric Synthetic Aperture Radar, Ph.D. Thesis, Ohio State University, 1993.

16. R. Dilsavor and R. Moses, "Fully-Polarimetric GLRTs for Detecting Scattering Centers with Unknown Amplitude, Phase, and Tilt Angle in Terrain Clutter," SPIE International Symposium on Optical Engineering in Aerospace Sensing, Orlando, FL, April 4-8, 1994.

17. L. Novak, Personal Communication, Summer 1994.

18. E. Kelly, "An Adaptive Detection Algorithm," IEEE Trans. on AES, Vol. 22, No. 1, March 1986, pp. 115-127.

19. E. Kelly and K. Forsythe, Adaptive Detection and Parameter Estimation for Multidimensional Signal Models, MIT Lincoln Laboratory Technical Report 848, 19 April 1989.

20. E. Anderson and I. Reed, " Adaptive Detection in SAR Using Multidimensional Signal Representations," Third Automatic Target Recognizer Systems and Technology Conference, Naval Postgraduate School, Monterey, CA, June 29-July 1, 1993.

21. E. Anderson, Adaptive Detection of Multidimensional Signals, Ph.D. Thesis, University of Southern California, August 1993.

22. X. Yu, L. Hoff, I. Reed, D. Buck, and A. Chen, "Comparative study of feature mapping and selection for ATR: experiments on SAR data," SPIE Conference on Algorithms for Synthetic Aperture Radar, Vol. 2230-32, Orlando, FL, April 67, 1994, pp. 275-284.

23. H.L. Van Trees, Detection, Estimation, and Modulation Theory, Part I, Wiley, New York, 1968.

24. C.J. Oliver, "Optimum texture estimators for SAR clutter," Journal of Physics D: Applied Physics, Vol. 26, 1993, pp. 1824-1835.

25. D.E. Kreithen, S.M. Crooks, W.W Irving, and S.D. Halversen, Estimation and Detection Using the Product Model, MIT Lincoln Laboratory Project Report STD-37, 19 March 1991.

26. V. Larson, Polarimetric subspace target detector for synthetic aperture radar data based on the huynen dihedral model, Dissertation, George Mason University, Summer 1995.

27. J.C. Henry, T.J. Murphy, and K.M. Carusone, "The Lincoln Laboratory millimeter-wave SAR imaging system," SPIE SAR Conference, Los Angeles, CA, January 1992. 